\title{
Getting to know the microbiome
}

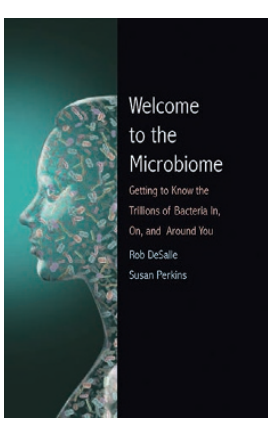

Welcome to the
Microbiome: Getting
to Know the Trillions
of Bacteria In, On
and Around You
by Rob DeSalle and
Susan Perkins
YALE UNIVERSITY PRESS:
2016. 288PP. US\$32.50

uman microbiome research is undergoing a renaissance, driven largely by advances in technologies such as 'next generation' sequencing that enable a deeper characterization of microbial populations than has ever been possible. Also underlying this renaissance is a paradigm shift in how microorganisms are viewed: transitioning from a focus on combatting pathogens to one that appreciates the importance of a complex interaction between polymicrobial communities and the host that has been evolving over millenia. In this book, Rob DeSalle and Susan Perkins of the American Museum of Natural History provide a guide to understanding the microbiome from an evolutionary perspective, giving an overview of not only the current state of understanding in the field, but also a history of how we got here.

The unique value of the book is the rich background it provides, including information on the microorganisms themselves (What are the major types? How are they named?), how we interact with microorganisms (How does the immune system function?), and the techniques used to study microorganisms (How do DNA sequences describe microbial communities?), all explored with an evolutionary/historical perspective. For instance, DeSalle and Perkins begin with the more existential question of 'What is life?', explaining the history of inquiry that informed our understanding of how life emerged and diverged over time to become the complex spectrum of life forms present today. When discussing the immune system, DeSalle and Perkins first describe microbialresistance mechanisms in plants and move into hydrozoans at the base of the animal branch of the tree of life, before ultimately addressing the incredible complexity of the immune system in humans. This progression illustrates to the reader how our complex relationship with microorganisms is the result of millennia of dynamic co-evolution. When discussing the methods for studying microorganisms, DeSalle and Perkins begin their account in the 1600s, when Robert Hooke and Antonie van Leeuwenhoek were first using microscopy to observe microorganisms (or 'animicules' as van Leeuwenhoek called them), proceeding to a description of how sequence-based 'culture-independent' techniques coupled with advances in throughput has catapulted our knowledge of microbial diversity forward.

Despite the rich history of microbial research, the relative infancy of microbiome research is highlighted. Much of the new research discussed includes observational studies that address fundamental questions: what types of microorganisms typically inhabit the diverse habitats our bodies provide? How variable is the microbiome across people, populations, and over time? How are human microbiomes acquired in early life and how do they change throughout life? How are microorganisms transferred between people and between local environments? Baseline information on human microbiome composition patterns provides a critical framework for understanding the complex interaction between the microbiome and various disease states. However, knowing the functional properties of microbiomes and how they directly influence host biology are ultimately the keys to harnessing rich information on the microbiome to promote health.

The book's discussion of the role the microbiome may play in health highlights a very important concept: it is not always straightforward to characterize a particular microorganism as 'good' or 'bad' or a particular microbiome state as 'healthy' or 'unhealthy'. A cited example is Helicobacter pylori - a species of bacteria that humans have been living in relative harmony with for at least 100,000 years, but that has been greatly reduced in prevalence due to improved hygiene and widespread antibiotic use. $H$. pylori has been considered a pathogen because its colonization of the stomach increases the risk for peptic ulcers and gastric cancer, but it turns out that $H$. pylori also protects against gastro-oesophageal reflux disease and linked disorders including oesophageal carcinoma. Clearly, even individual microorganisms need to be considered as a part of a complex system when trying to understand their relationship to health.
Finally, the book discusses how researchers are going beyond observational studies to establish a mechanistic link between the microbiome and particular diseases. For instance, they describe the importance of research using germ-free 'bubble mice' (that is, mice raised in sterile environments) to identify phenotypes caused by a lack of microorganisms. They continue with a discussion of how mouse models have allowed for the establishment of causal links between microorganisms and complex diseases such as obesity and depression/anxiety. However, this really only scratches the surface of the deluge of recent research on this topic - literature linking the microbiome with a myriad of allergic and autoimmune disorders, cardio-metabolic diseases and various cancers are not described, and the various mechanisms through which the microbiome can affect health could have benefited from a more thorough treatment.

Mechanistic understanding is essential for translating rich information on the complexities of the microbiome into advancements in human health. In a chapter addressing the question 'What are our defenses?', there is a discussion of our natural defences (our immune system), as well as human contrived defences such as vaccines and antibiotics, and how and why microorganisms can develop resistance. However, vaccines and antibiotics are both classes of therapeutics that fit neatly within the 'one pathogen one disease' paradigm. Some description of new research concerning 'beneficial bacteria' as potential therapeutics is given, but lacks a thorough treatment of how the complexity of interactions between microbiome and host may have the potential to inform 'next generation' therapies.

While this book would benefit from a deeper exploration of the radical advancements in understanding the microbiome's role in human health, the historical foundation it provides on the topic is excellent. Overall, this is a wonderful primer for anyone interested in learning more about the amazing world of unseen life that is teeming on and in our bodies and all around us.

REVIEWED BY CATHERINE A. LOZUPONE Catherine A. Lozupone is an assistant professor at the University of Colorado, Denver 80045, USA. e-mail: catherine.lozupone@ucdenver.edu 